\title{
A Review of Two Promising Radiosensitizers in Brain Metastases: Rrx-001 and 2-Deoxyglucose
}

\author{
Bryan T. Oronsky $^{1 *}$, Neil C. Oronsky ${ }^{2}$, Gary R. Fanger ${ }^{1}$, Arnold L. Oronsky³, Michelle M.C. Lybeck¹, Harry E. Lybeck ${ }^{4}$, Scott Z. Caroen ${ }^{1}$, \\ Christopher W. Parker ${ }^{1}$ and Jan Scicinski ${ }^{1}$
}

${ }^{1}$ EpicentRx Inc, 800 W El Camino Real, Suite 180, Mountain View, CA 94040, USA

${ }^{2}$ CFLS, LLC, 560 South Winchester Boulevard, San Jose, CA 95128, USA

${ }^{3}$ InterWest Partners, 2710 Sand Hill Road \#200, Menlo Park, CA 94025, USA

${ }^{4}$ Helsinki University, Yliopistonkatu 4, 00100 Helsinki, Finland

\begin{abstract}
The origin of the common phrase "your name is mud" may derive from the ordeal of 19th century physician, Dr. Samuel Mudd, who was perhaps wrongly convicted of conspiracy in the assassination of President Abraham Lincoln. Mudd's crime may have only been bad luck: Lincoln's assassin, John Wilkes Booth, allegedly previously unknown to the doctor, had broken his leg and happened across Mudd who, unwisely, as it turned out, set the fracture, and his own subsequent fate, which included life imprisonment with hard labor, making him a potential victim of circumstance rather than the perpetrator of a crime. Mudd's grandson, also a physician, tried unsuccessfully to clear his grandfather's widely reviled name, which as a result has remained, both literally and figuratively, Mudd.

This historical analogy highlights the important point that radiosensitizers as a class have been ignored rather than adored due to their failed reputation. Hence, in the field of radiation oncology, the "your name is mud" expression applies to radiation sensitizers, which from hyperbaric oxygen and the nitroimidazoles, to motexafin gadolinium, tirapazamine and efaproxiral have generally overpromised and under delivered with respect to survival treatment benefits in multiple different indications. However, newer non-toxic radiosensitizers on the horizon such as the antienergetic epigenetic redox modulator, RRx-001, that will start a Phase 2 clinical trial with concurrent whole brain radiotherapy (WBRT) in subjects with brain metastases, may finally validate the underlying promise and unrealized potential of these agents. The successful treatment of brain metastases is at least a four-hurdle process involving penetration, retention, selectivity and toxicity. This article will review the mechanism of the radiosensitizers, RRx001, and 2-deoxyglucose, as examples or "role models" for therapies that theoretically are able to overcome these substantial in vivo obstacles to successfully treat brain metastases.
\end{abstract}

It is the thesis of this review that new radiosensitizers are urgently needed and their poor reputation should be overcome.

Keywords: Radiosensitizers; Radiosensitization; Brain metastases; Epigenetics; RRx-001; 2-deoxyglucose

Abbreviations: WBRT: Whole Brain Radiotherapy; BMs: Brain Metastases; BBB: Blood Brain Barrier; CNS: Central Nervous System; ABC Proteins: ATP-Binding Cassette Proteins; 2-DG: 2-Deoxyglucose; NO: Nitric Oxide; ONOO-: Peroxynitrite; RBC: Red Blood Cell; SD: Stable Disease; RT: Radiotherapy

\section{Introduction}

The use of the umbrella term "brain metastases" categorizes different cancers under one rubric, as if they were all part of the same histologic group, when, in fact, what they share, as a class, is not histology but real estate: multiple tumor types metastasize to the brain, including most commonly lung cancer, breast cancer, melanoma, colon and kidney cancer $[1,2]$.

Brain metastases are the most frequent intracranial neoplasms in adults, with an estimated incidence in the USA of 200,000 cases per year, which exceeds that of primary brain tumors. Prognosis is generally poor to dismal owing to the dearth of effective treatment options, which is related at least in part to the physical impediment of the blood brain barrier, and survival is generally measured in months, approximately $4-6$, rather than years, after diagnosis. These poor clinical outcomes have prompted the Food and Drug Administration [3] to designate brain metastases as an unmet medical need.

\section{Existing Therapies}

Arguably, as systemic therapies have progressively improved, resulting in the control of extracranial metastases and longer life spans, clinically symptomatic brain metastases have proportionally increased [4] either due to the 'awakening' of long dormant tumor cells, sheltering behind the blood brain barrier (BBB) sanctuary or due to a higher overall cumulative frequency as patients are more likely over time to develop BM.

Whole brain radiotherapy (WBRT), stereotactic radiosurgery (either alone or as a "boost" to WBRT), and surgical resection are the mainstays of loco-regional treatment for BMs. Treatment strategies must balance possible clinical benefit against toxicities and reduction in the performance status of patients, while maintaining neurological function [5]. Their application may result in early and transient amelioration of symptoms, without increasing or prolonging survival. Furthermore the efficacy of treatment is limited by the acquired and

*Corresponding author: Bryan T. Oronsky, EpicentRx Inc, 800 W El Camino Real, Suite 180, Mountain View, CA 94040, USA, Tel: (408) 569 3202; Fax: 650-3964450; E-mail: boronsky@epicentrx.com

Received March 01, 2015; Accepted April 24, 2015; Published April 27, 2015

Citation: Oronsky BT, Oronsky NC, Fanger GR, Oronsky AL, Lybeck MMC, et al. (2015) A Review of Two Promising Radiosensitizers in Brain Metastases: Rrx-001 and 2-Deoxyglucose. J Cancer Sci Ther 7: 137-141. doi:10.4172/19485956.1000338

Copyright: @ 2015 Oronsky BT, et al. This is an open-access article distributed under the terms of the Creative Commons Attribution License, which permits unrestricted use, distribution, and reproduction in any medium, provided the original author and source are credited. 
intrinsic resistance of tumors [6]. While multiple mechansims of radioresistance have been proposed, such as enhanced DNA damage repair, upregulation of Antioxidant Response Element genes (ARE) and mutated p53 status [7], chief among them is hypoxia [8].

Well-oxygenated cells are more susceptible to the cytotoxic effects of radiation than their hypoxic counterparts. The oxygen enhancement ratio, described as the relative sensitivity of oxic cells/anoxic cells to the lethal effects of low Linear-Energy-Transfer (LET) radiation, typically ranges between 2.5 and 3.0. Oxygen promotes free radical formation. Irradiation results in the radiolysis of water: the initial formation of an ion radical forms the highly reactive hydroxyl radical after reaction with another water molecule. In the presence of oxygen peroxide is formed after reaction with the hydroxyl radical, resulting in "fixation" or permanent cellular and DNA damage [8].

In the absence of oxygen, peroxide is not formed: sulfhydrylcontaining groups such as cysteine and glutathione reconstitute DNA through hydrogen donation. As a result hypoxia is the chief culprit of radioresistance both due to increased free radical scavenging and upregulation of the transcription factor HIF-1a, which increases the malignant potential of tumors leading to more aggressive survival traits and resistance to radiation [8]

\section{Radiosensitizers}

Radiosensitizers can be characterized as chemical or pharmacological agents used in combination with radiation to increase its efficacy. Studies of existing radiosensitizers in combination with WBRT have shown little improvement of outcomes. Tsao et al. [9], summarizing the results of five randomized controlled trials that examined the use of radiosensitizers in addition to WBRT, concluded that no benefit in terms of overall survival or brain response was found [10].

Given the importance of radiotherapy, candidates such as motexafin gadolinium (Xcytrin), Efaproxyn (efaproxiral or RSR13) and bortozemib (Velcade) as well as thalidomide, teniposide, topotecan, paclitaxel, and cisplatin, have all been administered in combination with WBRT in brain metastases, and none have demonstrated enough of a radiosensitization benefit to risk ratio to support their routine use. The efficacy of radiotherapy is further limited by the intrinsic or acquired radioresistance of cancer cells [11]. As a class, then, figuratively speaking, the name of radiosensitizers, in brain metastases is mud.

Clearly due to the lack of clinical benefit and the prevalence of radiotherapy to treat brain metastases, an urgent unmet need for new radiosensitizers exists. New radiosensitizers need to possess the following four attributes; presented below.

The first sine qua non in the treatment of brain metastases is penetration of the blood brain barrier.

The central nervous system (CNS) is an immunologically privileged site, and even though cancer-induced inflammation may physically disrupt the blood brain barrier, the delivery and access of large and small molecular therapeutics [12] is still impeded as a result of osmotic pressure and enzymatic degradation. The BBB is a physical [13] and a metabolic [14] barrier: tight junctions between capillary endothelial cells formed through cell adhesion molecules physically exclude polar ionic molecules while enzymes such as peptidases, nucleotidases, monoamine oxidase and cytochrome P450 serve to metabolize and inactivate xenobiotic substances that manage to surmount the BBB. In cancer the disruption of the blood brain barrier, which in theory should benefit the transport and delivery of anticancer agents, is counteracted by cerebral edema and increased interstitial fluid pressure due to fluid seepage from leaky vessels [15], that results in even less permeability than normal brain endothelium (Figure 1).

A second sine qua non condition is retention; transporter ATPbinding cassette $(\mathrm{ABC})$ proteins [16], present at the $\mathrm{BBB}$ interfaces, actively efflux compounds from the brain and prevent or diminish accumulation.

The third and fourth conditions, which are related, involve selectivity and toxicity since selective and effective localization of anticancer therapies in the tumor prevents unintended toxicity to normal tissues.

Due to these multiple layers of "armor" which protect and support the normal brain as well as provide a safe haven for metastases, the clinical landscape is generally lacking in effective therapeutic options (Figure 2); emerging radiosensitizers for the treatment of brain metastases include RRx-001, the epigenetic nitro-oxidative modulator with anti-energetic properties, and 2-deoxyglucose (2-DG), the energy restriction mimetic agent, both briefly reviewed below.

\section{RRx-001}

$\mathrm{RRx}-001$, a potent nitric oxide donor and inhibitor of 3 epienzymes, histone deacetylases, DNA methyltransferases and lysine

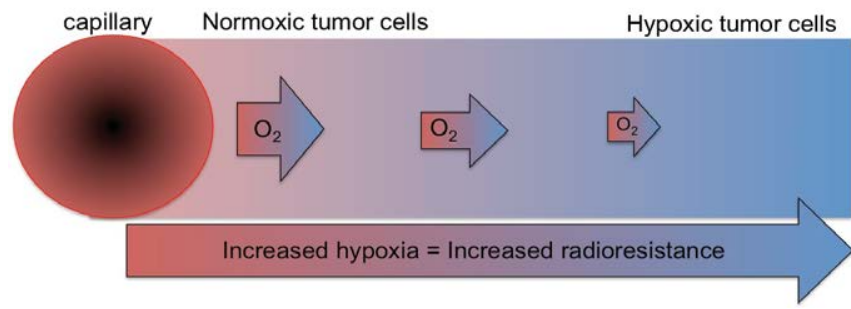

Figure 1: Tumor hypoxia and radioresistance: as the distance from the peripheral vasculature increases, chronic hypoxia also increases due to the consumption of oxygen by actively respiring tumor cells. Hypoxia is correlated with radioresistance because oxygen radicals fix tumor DNA damage. If oxygen is not present, sulfhydryl containing molecules restore or reconstitute the free radical damage to DNA.

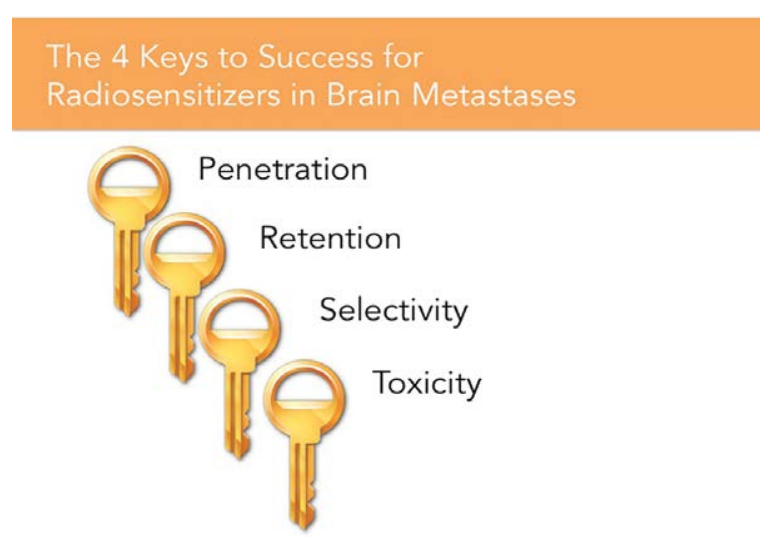

Figure 2: The four keys to success for radiosensitizers in brain metastases, which historically have been characterized by a paucity of viable therapeutic options due to an inability to overcome at least four treatment hurdles. In theory these keys or criteria for success in brain metastases are met by RRx001 and 2-DG. 
demethylases, in Phase 2 studies as an anti-cancer agent, is both a radiosensitizer and resensitizer of chemorefractory tumors $[17,18]$. A Phase $1 \mathrm{~b} / 2 \mathrm{a}$ clinical trial of RRx-001 and whole brain radiotherapy (WBRT) in brain metastases is planned at the University of Michigan under the aegis of Drs. Kim, Lao and Lawrence.

In the pro-oxidant environment of the tumor and its environs $\mathrm{RRx}-001$ bound hemoglobin is stimulated to overproduce nitric oxide, which oxidizes the intracellular milieu of the red blood cell [19], leading to the release of heme and LDL-containing microvesicles from the RBC membrane (EpicentRx unpublished data). According to Lakhal and Wood [20], microvesicles can bypass the blood brain barrier, which would amplify RRx-001-induced oxidative stress and synergize with radiation (Figure 3 ).

\section{1st barrier: penetration}

The BBB or inflammation-related structural/osmotic impediments to the movement of ions, polar solutes, and macromolecules have no impact on the free diffusion of gases such as oxygen and nitric oxide. The permeability to nitric oxide gas mediates the therapeutic activity of $\mathrm{RRx}-001$ in infectious disease indications such as cerebral malaria and theoretically in brain metastases.

RRx-001 covalently binds to specific intracellular antioxidants and a conserved residue on hemoglobin, $\beta$ Cys 93 , which increases hemoglobin-oxygen affinity, presumably facilitating oxygen unloading to deeply hypoxic tissues. In addition, allosteric regulation of nitrite reduction by deoxyhemoglobin through binding of $\mathrm{RRx}-001$ to $\beta$ Cys 93 catalyzes the superproduction of nitric oxide (NO) during hypoxia, which also likely favors increased oxygenation and blood flow to tumors. At these high concentrations, NO, which readily diffuses across the blood brain barrier, reacts with superoxide anion, generated at higher

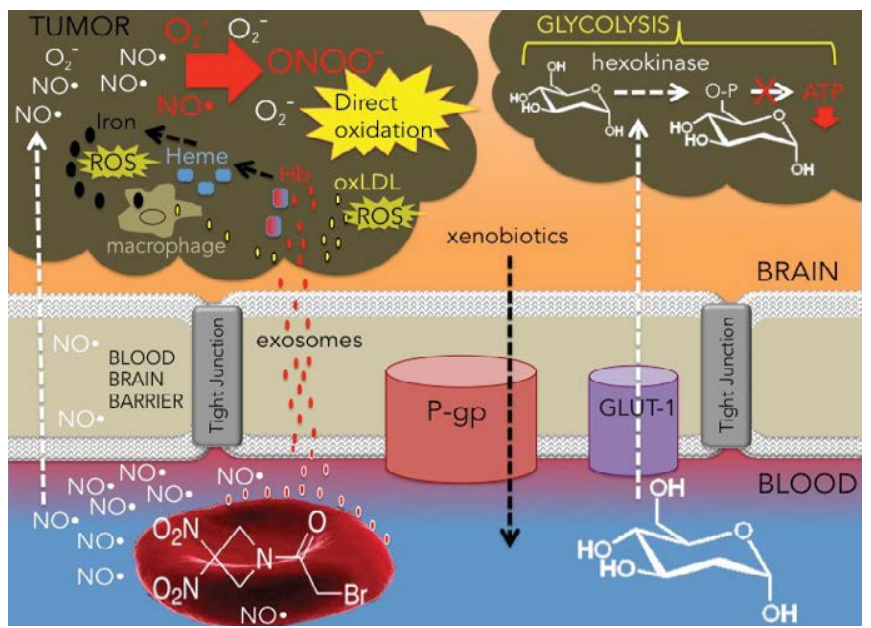

Figure 3: In this illustration, on the left, the RRx-001-bound red blood cell is depicted as a 'catalytic converter' of nitrite to nitric oxide under prooxidant conditions in the vicinity of the tumor. As a free radical gas, nitric oxide a) diffuses through the blood brain barrier and combines with endogenously elevated levels of superoxide in the tumor to produce the potent biological oxidant peroxynitrite (ONOO-) b) oxidizes the internal milieu of the red blood cell (RBC) resulting in the shedding of heme and LDL-containing microvesicles, theoretically passing the blood brain barrier and amplifying oxidative stress in the tumor. On the right, 2-deoxyglucose, which is taken up by GLUT-1, is a substrate for hexokinase but not phosphoglucose isomerase so 2-deoxyglucose-6-phosphate accumulates in the cell, inhibits hexokinase and reduces glycolytic flux, resulting in ATP depletion. The ATP-dependent P-gp pump, responsible for the efflux of xenobiotics across the BBB, is also depicted. levels in tumors, to produce peroxynitrite (ONOO-), a 'super' oxidizing agent, which mediates various effects including irreversible inhibition of glycolysis, mitochondrial respiration, epigenetic 'reactivation' of silenced tumor suppressor genes and DNA fragmentation. As a blood flow, oxidative stress and hemoglobin modulator, RRx-001 has the potential to eliminate deeply hypoxic radioresistant cells,thus enhancing the effects of radiotherapy (RT).

\section{2nd barrier: retention}

Since the parent drug itself reacts with red blood cells, and stimulates them to produce nitric oxide, which, as a gas, is not a substrate for the efflux pumps, retention is not an issue.

\section{3rd and 4th barriers: selectivity and toxicity}

By themselves nitric oxide and superoxide are relatively benign and poorly reactive until they combine to produce the more toxic and detrimental species, hydrogen peroxide, $\mathrm{H}_{2} \mathrm{O}_{2}$, and peroxynitrite, -OONO [21]. Tumors are characterized by higher levels of oxidative stress $\left(\mathrm{H}_{2} \mathrm{O}_{2}\right.$ and superoxide) than normal tissue, which results in the preferential generation of the highly reactive peroxynitrite in the presence of high levels of nitric oxide [22], thus accounting for the specificity and decreased toxicity of RRx-001 treatment.

In the Phase 1 first-in-human single agent clinical trial, twentyfive subjects were treated with a weekly or biweekly iv infusion at dose levels of $10,16.7,24.6,33,55,18$ and $83 \mathrm{mg} / \mathrm{m}^{2}$. With the exception of one subject, treatment related toxicities were limited to grade $\leq 2$. Localized infusional pain was most common (92\%), while all other toxicities were reported in $<10 \%$ of subjects. The dose-limiting toxicity was infusional pain, resulting from local NO release, rather than from any deleterious effects on other organ systems, and while a maximum tolerated dose was not reached, the current maximum feasible dose was determined as $83 \mathrm{mg} / \mathrm{m}^{2}$ using the current infusion rates at the present time. The selected Phase 2 doses, $10-16.5 \mathrm{mg} / \mathrm{m}^{2}$, were both pharmacodynamically active and relatively well tolerated in the Phase 1 study.

One objective response was observed but in the majority (71\%) of subjects clinical benefit manifested as stable disease (SD) at 8 weeks or longer in a variety of tumor types including lung cancer, which accounts for approximately one half of all brain metastases. One subject with prolonged SD ( $\sim 10$ months) received palliative radiotherapy (RT) in fractions at a variety of locations; a rapidly progressing, mitotically active clavicular metastasis turned stably PET negative, indicating complete necrosis while the other lesions improved in terms of a decrease in pain without any evidence of potentiation of the radiation effect on normal tissues. These Phase 1 results indicate that RRx-001 is safe and tumor selective, since the only normal tissue toxicity was transient infusion site pain.

In summary, the rationale to study RRx-001 in brain metastases with RT in an upcoming Phase 2 clinical trial is essentially two-fold: 1) as a single agent $\mathrm{RRx}-001$ was broadly active against a range of tumor types 2) nitric oxide, an intrinsic radiosensitizer, is induced by RRx001 via deoxyhemoglobin and, as a gas, is able to freely diffuse across membranes such as the blood-brain barrier potentially to radiosensitize hypoxic tumor cells.

\section{2-DG}

2-deoxyglucose is a non metabolizable glucose analogue that acts as a competitive inhibitor of glycolysis [23,24]. 2-DG takes advantage of glucose uptake through the blood brain barrier via the 
glucose transporter, GLUT-1. Upon intracellular transport, 2-DG is phosphorylated by the glycolytic enzyme, hexokinase, to 2-DG-P, which is a poor substrate for phosphohexose isomerase. As a result, 2-deoxyglucose-P is trapped and accumulates in cells, similar to the irreversible trapping of (18F)luorodeoxyglucose, used for PET scans. Selective inhibition of hexokinase activity by 2-DG interferes with ATP formation and leads to tumor cell death [25-27].

\section{1st barrier: penetration}

Since the brain is critically dependent on glucose as an energy substrate, 2-DG, as an analogue of glucose, is taken up quickly through the BBB.

\section{2nd barrier: retention}

Because of the avid accumulation of glucose by tumor cells as a result of a greater reliance on glycolysis for energy production, 2-DG, like the fluorodeoxyglucose tracer in PET imaging, accumulates in tumors through the GLUT transporters, where it competitively interferes with glucose metabolism [28].

\section{3rd and 4th barriers: selectivity and toxicity}

The altered tumor metabolism predisposes to preferential localization of 2-DG within the tumor, with relatively selective destruction of the tumor. In terms of toxicity, however, while 2-DG generally spares normal cells, it is associated with Q-T prolongation; in a recent Phase 1 trial of $2 \mathrm{DG}$ in combination with docetaxel, in patients with advanced solid tumors the most significant adverse effects were reversible hypoglycemia and reversible grade 3 QTc prolongation [29]. Since the heart is dependent upon the uptake of extracellular glucose under conditions of oxygen deprivation, cardiac performance is adversely affected without exogenous glucose under oxygen-limiting conditions. In a Phase $1 / 2$ trial in cerebral gliomas with large fraction radiotherapy (5 Gy) 2-DG was well tolerated and enhanced the effects of radiotherapy.

In summary, the rationale to study 2-DG in brain metastases with RT is also essentially two-fold: 1 ) as a single agent $2-D G$ is preferentially and selectively taken up by the BBB and retained by tumor cells. Interference with glucose metabolism will impair antioxidant production and sensitize cancer cells to radiation 2). Although it is associated with cardiac side effects, 2-DG is generally well tolerated.

\section{Conclusion}

For a non-rare, non-orphan disease, with a bleak prognosis and an incidence that is estimated at 200,000 patients per year in the US, brain metastases are in general underserved, understudied and marginalized; this is a therapeutic arena where progress and innovation have taken a backseat to stagnation and treatment is limited to a few unsatisfactory options; to add insult to injury, most subjects are actively and routinely excluded from clinical trials, which are skewed toward nonCNS disease. Brain metastases are historically poorly responsive to chemotherapy, a function of limitations imposed by uptake, retention, selectivity and toxicity.

It would appear from the Phase 1 trial that $\mathrm{RRx}-001$ is broadly active in a myriad of tumor types, possibly even 'histology agnostic', systemically well-tolerated and safe, perhaps making it ideally suited to treat a population of all-comers in brain metastases. In addition, the RRx-001-induced production of NO, a soluble free radical gas, as well as lipid and iron-laden microvesicles, are anticipated to diffuse towards bystander tumor cells, resulting in single agent activity as well as radiosensitization.
While no formal evaluation of RRx-001 as a radiosensitizer has been conducted in humans, the preclinical evidence and Phase 1 results suggest given its apparent indiscriminate and non-selective cytotoxicity with regard to tumor type, synergistic interaction with ionizing radiation and potential radioprotective properties, and lack of systemic toxicities due to a selectivity for malignant cells, that RRx-001 has the potential to treat the wide range of tumors which constitute brain metastases with minimal systemic toxicity.

Similarly, 2-deoxyglucose is systemically well tolerated and crosses the blood brain barrier where it is taken up by metabolically active tumor cells. A Phase 1/2 clinical trial in malignant gliomas suggests a radiosensitizing effect of 2-DG.

It is the thesis of this mini-review that successful radiosensitizers in the treatment of brain metastases must overcome the hurdles for penetration, retention, selectivity and toxicity like RRx-001 and 2-DG.

If they are successful, as hoped for and anticipated, this new generation of radiosensitizers at the vanguard will have the opportunity, unlike the namesakes of Dr. Samuel Mudd, to successfully restore and evolve the reputation of radiosensitizers as a whole.

\section{Acknowledgements}

The authors acknowledge Dr. Ted Lawrence at the University of Michigan who had the insight-and hopefully the inspiration-to suggest a clinical trial with RRx-001 in brain metastasis and bring together a team of exceptionally bright and curious clinicians and Ph.D.s including Drs. Kim, Lao, Mammoser, Schipper, and Cao to make it happen. We patiently look forward to working with you.

\section{References}

1. Nieder C, Spanne O, Bilberg I, Dalhaug A (2010) Primary Tumour Characteristics as Potential Prognostic Factors in Brain Metastases from Breast Cancer. J Cancer Sci Ther 2: 070-073.

2. American Brain Tumor Association: Metastatic Brain Tumors, 2015.

3. Yang H, Lee HW, Kim Y, Lee Y, Choi YS, et al. (2013) Radiosensitization of brain metastasis by targeting c-MET. Lab Invest 93: 344-353.

4. Elaimy AL, Demakas JJ, Mackay AR, Lamoreaux WT, Fairbanks RK, et al. (2010) Treatment of Brain Metastases: Past, Present and Future Directions. J Nucl Med Radiat Ther S2:002.

5. Islamian JP, Hatamian M, Rashidi MR (2015) Nanoparticles promise new methods to boost oncology outcomes in breast cancer. Asian Pac J Cancer Prev 16: 1683-1686.

6. Williams JR, Gridley DS, Slater JM (2011) Radiobiology of Radioresistant Glioblastoma, Advances in the Biology, Imaging and Therapies for Glioblastoma. Prof. Clark Chen (Ed.), ISBN: 978-953-307-284-5, InTech.

7. Oronsky BT, Knox SJ, Scicinski J (2011) Six degrees of separation: the oxygen effect in the development of radiosensitizers. Transl Oncol 4: 189-198.

8. Tsao MN, Lloyd NS, Wong RK, Rakovitch E, Chow E, et al. (2005) Radiotherapeutic management of brain metastases: a systematic review and meta-analysis. Cancer Treat Rev 31: 256-273.

9. Viani GA, Manta GB, Fonseca EC, De Fendi LI, Afonso SL, et al. (2009) Whole brain radiotherapy with radiosensitizer for brain metastases. J Exp Clin Cancer Res 28: 1.

10. Cui FB, Liu Q, Li RT, Shen J, Wu PY, et al. (2014) Enhancement of radiotherapy efficacy by miR-200c-loaded gelatinase-stimuli PEG-Pep-PCL nanoparticles in gastric cancer cells. Int J Nanomedicine 9: 2345-2358.

11. Pardridge WM (2005) The blood-brain barrier: bottleneck in brain drug development. NeuroRx 2: 3-14.

12. Wolburg $H$, Lippoldt A (2002) Tight junctions of the blood-brain barrier development, composition and regulation. Vascul Pharmacol 38: 323-337.

13. el-Bacha RS, Minn A (1999) Drug metabolizing enzymes in cerebrovascular endothelial cells afford a metabolic protection to the brain. Cell Mol Biol (Noisyle-grand) 45: 15-23. 
Citation: Oronsky BT, Oronsky NC, Fanger GR, Oronsky AL, Lybeck MMC, et al. (2015) A Review of Two Promising Radiosensitizers in Brain Metastases: Rrx-001 and 2-Deoxyglucose. J Cancer Sci Ther 7: 137-141. doi:10.4172/1948-5956.1000338

14. Gerstner ER, Duda DG, di Tomaso E, Ryg PA, Loeffler JS, et al. (2009) VEGF inhibitors in the treatment of cerebral edema in patients with brain cancer. Nat Rev Clin Oncol 6: 229-236.

15. Abbott NJ, Rönnbäck L, Hansson E (2006) Astrocyte-endothelial interactions at the blood-brain barrier. Nat Rev Neurosci 7: 41-53.

16. Oronsky BT, Knox SJ, Scicinski JJ (2012) Is Nitric Oxide (NO) the Last Word in Radiosensitization? A Review. Transl Oncol 5: 66-71.

17. Reid T, Dad S, Korn R, Oronsky B, Knox S, et al. (2014) Two Case Reports of Resensitization to Previous Chemotherapy with the Novel Hypoxia-Activated Hypomethylating Anticancer Agent RRx-001 in Metastatic Colorectal Cancer Patients. Case Rep Oncol 7: 79-85.

18. Fens M, Sandra KL, Claudia RM, Bill F, Jan S, et al. (2011) NO or no NO, increased reduction of nitrite to nitric oxide by modified red blood cells. Blood (ASH Annual Meeting Abstracts) 118: 2125.

19. Lakhal S, Wood MJ (2011) Exosome nanotechnology: an emerging paradigm shift in drug delivery: exploitation of exosome nanovesicles for systemic in vivo delivery of RNAi heralds new horizons for drug delivery across biological barriers. Bioessays 33: 737-741.

20. Afanas'ev I (2010) Signaling and Damaging Functions of Free Radicals in Aging-Free Radical Theory, Hormesis, and TOR. Aging Dis 1: 75-88.

21. Chirico EN, Pialoux V (2012) Role of oxidative stress in the pathogenesis of sickle cell disease. IUBMB Life 64: 72-80.

22. Aghaee F, Pirayesh Islamian J, Baradaran B (2012) Enhanced radiosensitivity and chemosensitivity of breast cancer cells by 2-deoxy-d-glucose in combination therapy. J Breast Cancer 15: 141-147.

23. Ahmad IM, Mustafa EH, Mustafa NH, Lubana HT, Maher YA (2010) 2DG enhances the susceptibility of breast cancer cells to doxorubicin. Cent Eur $\mathrm{J}$ Biol 5: 739-748.

24. Kim JW, Dang CV (2006) Cancer's molecular sweet tooth and the Warburg effect. Cancer Res 66: 8927-8930.

25. Calvo MB, Figueroa A, Pulido EG, Campelo RG, Aparicio LA (2010) Potential role of sugar transporters in cancer and their relationship with anticancer therapy. Int J Endocrinol 2010.

26. Oronsky BT, Oronsky N, Fanger GR, Parker CW, Caroen SZ, et al. (2014) Follow the ATP: tumor energy production: a perspective. Anticancer Agents Med Chem 14: 1187-1198.

27. Guo J, Du C, Shan L, Zhu H, Xue B, et al. (2012) Comparison of near-infrared fluorescent deoxyglucose probes with different dyes for tumor diagnosis in vivo. Contrast Media Mol Imaging 7: 289-301.

28. Raez LE, Papadopoulos K, Ricart AD, Chiorean EG, Dipaola RS, et al. (2013) A phase I dose-escalation trial of 2-deoxy-D-glucose alone or combined with docetaxel in patients with advanced solid tumors. Cancer Chemother Pharmacol 71: 523-530.

29. Mohanti BK, Rath GK, Anantha N, Kannan V, Das BS, et al. (1996) Improving cancer radiotherapy with 2-deoxy-D-glucose: phase I/II clinical trials on human cerebral gliomas. Int J Radiat Oncol Biol Phys 35: 103-111. 